\title{
Elles se relèvent : penser la résurgence dans la langue et la littérature Innues
}

\author{
Marie-Ève Bradette \\ Université de Montréal
}

La mémoire se transmet par le sang. Mémoire écorchée, démembrée, violée. Mémoire effacée de la conscience du peuple. Un grand vide se creuse d'une génération à l'autre. Lorsque le récit n'est pas raconté, il y a privation.

- Natasha Kanapé Fontaine 
Depuis le début des années 2000, les écrivaines Innues ${ }^{1}$ se positionnent à l'avant-scène de la production littéraire du Québec et, de ce fait, travaillent à même le vide et la privation pour donner à lire un récit tantôt intime, tantôt collectif : celui de leur culture, de leur nation, une histoire autrement écorchée, effacée, un « récit qu'on ne raconte pas » (Bacon, 2009, p. 82). Parmi ces écrivaines, on pense bien évidemment à Joséphine Bacon, Natasha Kanapé Fontaine, Naomi Fontaine et Marie-Andrée Gill, mais aussi à des autrices moins connues, par exemple à Mélina Vassiliou, Maya Cousineau-Mollen, Alyssa Jérôme, Manon Nolin et, plus récemment encore, à J.D. Kurtness qui toutes ont publié au moins un livre depuis les dix dernières années. Ces femmes, et même Joséphine Bacon dont les premiers textes sont pourtant publiés dans des revues littéraires dès la fin des années 1970, mais dont le premier recueil de poésie paraît seulement en 2009, sont considérées comme prenant part à une véritable émergence littéraire (Caron, 2012), un terme qui fait d'ailleurs bien rire Joséphine Bacon (Tardif, 2018). Dans son article «La plume autochtone / Émergence d'une littérature », Jean-François Caron emploie également l'expression d'une « littérature neuve » (2012, p. 12) pour décrire les littératures autochtones dans le contexte francophone du Québec. Toutefois, dire des écritures autochtones qu'elles sont émergentes c'est opérer a négation de pratiques littéraires (orales comme scripturaires²) plus anciennes. Au contraire, reléguer ses pratiques actuelles à une littérature dite «traditionnelle» occulterait la

\footnotetext{
${ }^{1}$ Tout au long de cet article, si je place le mot «Innu » systématiquement avec une lettre majuscule c'est pour insister, comme le fait et le propose Daniel Heath Justice dans son récent essai Why Indigenous Literatures Matter (2018, p. 6), et dans la matérialité même de mon énonciation, sur la singularité culturelle et politique d'un peuple dont l'emploi adjectival du substantif ne permet pas de rendre compte.

${ }^{2}$ Au sujet de la conception d'une littérature orale qui précéderait la littérature autochtone écrite en tant qu'elle participe de la même tradition que cette dernière et, dès lors, permet de les constituer toutes deux en une seule et même histoire littéraire, l'ouvrage de Diane Boudreau (1993) compte encore aujourd'hui parmi les textes fondateurs de la critique littéraire au sujet des littératures autochtones du Québec.
} 
MARIE-ÈVE BRADETTE, «Elles se relèvent : penser la résurgence dans la langue et la littérature Innues »

contemporanéité des enjeux et de la poétique des textes. Ainsi, à l'encontre de cette idée d'une littérature émergente, voire même d'une renaissance littéraire comme un mouvement semblable fut nommé dans le contexte de la littérature autochtone de la décennie 1970 aux États-Unis (Lincoln, 1985), je propose de réfléchir à la littérature Innue au féminin en tant qu'elle constitue une pratique de la résurgence inscrite, c'est là une caractéristique du concept même, dans un mouvement littéraire et culturel transgénérationnel. Une telle lecture des œuvres implique dès lors de prendre en considération la continuité transmise dans et par les textes de manière à analyser non seulement les ruptures, mais surtout les désirs (Tuck, 2009), les survivances (Vizenor, 1994 et 2009), les filiations et les traces de résurgence (Simpson, 2011) dans les textes de ces écrivaines. Plutôt que d'aborder les enjeux d'une littérature émergente, je propose ainsi de réfléchir, pour reprendre les termes de Leanne Betasamosake Simpson, à une «nouvelle émergence », à une résurgence de la pensée et de la littérature Innues et d'y réfléchir à partir de la méthodologie proposée par l'autrice de Dancing on our Turtle's Back (Danser sur le dos de notre Tortue) pour qui la langue, en ce qui la concerne le nishnaabemowin, et l'ensemble des savoirs contenus en elle devient une méthode de recherche et d'analyse (Simpson, 2018 [2011]). Or, ce que je propose ici, plutôt que de réfléchir à la langue dans ses seuls aspects sociolinguistiques, c'est d'aborder le langage littéraire comme un prisme de conceptualisation. Ce faisant, ce n'est pas seulement la langue Innue qui sera considérée, mais aussi la langue française et ses possibilités ou impossibilités d'exprimer la complexité culturelle Innue. En ce sens, le langage littéraire fait se télescoper les langues Innue et française en un matériau créateur singulier.

Nonobstant, pour bien comprendre ce que signifie utiliser le langage comme une méthode, puis le concept de résurgence central à la présente réflexion, il faut revenir à Leanne 
Betasamosake Simpson. En effet, l'autrice utilise le verbe biskaabiiyang pour aborder les termes d'une résurgence culturelle, pour la conceptualiser. Biskaabiiyang signifie tantôt « regarder en arrière », tantôt « retour sur nous-mêmes », mais est aussi entendu, par des locuteurs de la langue nishnaabeg (nishnaabemowin), comme un processus individuel et collectif de décolonisation (Simpson, 2018, p. 57-58). Dans tous les cas, il y a un retour, un regard posé vers ce qui est derrière, mais une vision qui s'enrichit du passé pour se tourner en direction de l'avenir, le créer, le recréer. Selon l'intellectuelle Nishnaabeg, «le biskaabiyang est donc une manière nishnaabe très utile et importante d'ancrer la résurgence ou la décolonisation comme une nouvelle émergence puisqu'elle est porteuse d'une signification importante et résonne à travers tout notre territoire. » (2018, p. 61. L'autrice souligne.) En choisissant ce mot pour élaborer une théorie résolument décoloniale, Leanne Betasamosake Simpson fait de la langue une intervention critique, une méthode, en somme, avec le projet « [d']approfondir notre compréhension de la décolonisation, de l'assimilation, de la résistance et de la résurgence depuis l'intérieur des points de vue nishnaabeg. » (2018, p. 57)

Glen Sean Coulthard nous aide, lui aussi, à définir, dans le champ des études autochtones (notamment dans le contexte anglophone du Canada), le concept de résurgence. Pour Coulthard, la résurgence est une alternative critique à la politique de la reconnaissance conceptualisée par Hegel, puis Fanon. Plutôt que d'aspirer à une reconnaissance institutionnelle, politique et économique qui implique nécessairement des rapports de pouvoir inégaux, les peuples autochtones doivent travailler à des pratiques de résurgence qui prennent racine dans des mouvements de résistance (Coulthard, 2018) et d'autodétermination. Pour le politicologue Déné, le mouvement Idle no More (qui notons-le 
MARIE-ÈVE BRADETTE, «Elles se relèvent : penser la résurgence dans la langue et la littérature Innues »

est un mouvement militant inauguré par des femmes autochtones dans l'ouest du Canada), est un exemple manifeste de ce qu'est et doit être une pratique localisée et effective de la résurgence. En conclusion de son ouvrage Peau rouge, masques blancs (Red skin, white mask), Coulthard se questionne sur la possibilité d'envisager la résurgence non seulement comme une pratique de déconstruction de la pensée coloniale, mais aussi comme une forme créatrice qui vise à faire advenir de nouveaux modèles politiques et économiques fondés sur la relation réciproque qu'entretiennent les premiers peuples avec le territoire (2018, p. 283). Ici, la réflexion de Coulthard s'appuie explicitement sur celle de Leanne Betasamosake Simpson qui, pour l'auteur de Peau rouge, masques blancs, compte parmi les premières, aux côtés de l'intellectuel Mohawk Gerald Taiaiake Alfred, à avoir étayé le concept.

En somme, dans le présent article et en prenant comme cadre théorique l'œuvre essayistique de Leanne Simpson, mais aussi en empruntant à sa méthodologie, j'aborderai la résurgence avec et dans le langage tel qu'il est mis en scène par la littérature, puis la langue Innue, une langue qui, et nous le verrons précisément avec les autrices, porte en elle le savoir du territoire, sa relation avec le monde naturel. En conséquence, l'objectif de cet article est double. Premièrement, il s'agira de formuler un cadre conceptuel à même de rendre compte de la complexité et de la spécificité des écritures autochtones, en général, et Innues, en particulier, et d'ainsi permettre de réviser les discours autour de l'idée d'une émergence littéraire dont les termes mêmes sont inappropriés. Pour ce faire, je considérerai l'idée d'une « relève » (du) littéraire dans ses aspects pluriels comme un geste langagier travaillé par la résurgence, qui travaille à son élaboration. Le second objectif, qui découle directement du premier, est de montrer que l'analyse des textes de certaines des autrices mentionnées résulte en la démonstration que cette conception d'une pratique littéraire à travers les générations, 
Www.revue-analyses.org, vol. 14, nº1, printemps- été 2019, p. 98-123.

une pratique qu'il convient d'examiner en guise de cadre critique, est interne aux œuvres

littéraires. À cette pratique transgénérationnelle et à sa mise en scène dans l'écriture s'ajoute, dans plusieurs textes du corpus Innu, un travail esthétique notable autour de l'idée même de la possibilité de «se relever », d'une relève dans le langage créatif et dans la langue, dans l'écriture et dès lors de faire advenir, à même l'articulation d'une posture debout, une création nouvelle, une re-création qui participe d'un processus de décolonisation en faisant du texte un espace éminemment politique. Ainsi, ce qui m'intéresse c'est la manière dont les écrivaines Innues mettent en scène, travaillent, compliquent et complexifient les concepts d'héritage transgénérationnel, de désir, de survivance et de résurgence, faisant de leur espace d'énonciation littéraire, un véritable discours théorique et politique tout à la fois. C'est donc en analysant les œuvres littéraires qu'il est possible de formuler un cadre conceptuel depuis lequel aborder les épistémologies contenues à même les littératures autochtones, des épistémologies qui sont façonnées par le rapport au langage, par la manière dont l'innuaïmun, par exemple, travaille à complexifier l'écriture en langue française, en langue coloniale. Ainsi, si je pars de la conception de la résurgence selon Leanne Betasamosake Simpson, c'est moins pour l'appliquer telle une grille d'analyse aux œuvres des autrices Innues que pour voir comment le concept de résurgence résonne avec la manière dont les femmes Innues se relèvent au cœur même de leurs interventions littéraires.

\section{Se relever dans l'écriture et dans la langue}

Dans son prologue à Bleuets et abricots, Natasha Kanapé Fontaine écrit : «Un cri s’élève en moi et me transfigure. Le monde attend que la femme revienne comme elle est née : femme debout, femme puissance, femme résurgence. Un appel s'élève en moi et j’ai décidé de dire oui à ma naissance. » (2016, p. 7) Ces quelques lignes donnent à lire une posture et un 
MARIE-ÈVE BRADETTE, «Elles se relèvent : penser la résurgence dans la langue et la littérature Innues »

mouvement dans ses rapports au féminin, à la femme qui, sujet de l'écriture, doit se relever à même le territoire imaginaire des mots, de la poésie. Elle doit se relever, revenir, faire retour, donc, faisant jouer ce même « re » mis en scène significativement par le verbe relever. Mais, plus directement encore, les mots de la poète Innue indiquent la posture debout de la femme, de la femme qui est à la fois puissance et résurgence. Par la mise en relation de ces différents mots, l'autrice donne le ton à sa poésie en même temps que ses clés de lecture. Puis, le prologue se poursuit :

Je suis parce que je suis. Je dis je. Je sais donner la vie. Je suis féconde. Le poème entre en moi comme un amant. L'univers entre en mon corps afin de continuer le mouvement du cycle vital. Tout est cercle. La terre. Les bleuets et les abricots. Le poème est le mouvement qui féconde.

Je suis le poème de l'existence.

Je sens tout. Les mémoires. Les blessures. Je vois tout. Le choc de la dépossession. Prendre la parole pour soulager le fardeau. Le poids de la douleur. J'écris pour dire oui. À moi. Femme.

Forcer les portes du silence. Assurer la trace. Redonner vie aux ombres, aux enfants brisés, à la parole qui ne sait plus dire oui. Qui ne sait plus se tenir. Qui ne sait plus tenir parole. (Kanapé Fontaine, 2016, p. 8)

Ici, Natasha Kanapé Fontaine thématise et représente ce qu'est le sens même d'une relève littéraire autochtone qui ne doit pas être comprise comme l'avènement d'une nouvelle ère de production littéraire rompant avec les œuvres antérieures, mais plutôt comme un espace de ré-écriture et de re-création informée de l'histoire, des textes, des traumatismes, des blessures, des désirs aussi, de sorte à relever la parole, à lui redonner, à cette parole féminine, sa posture verticale, sa possibilité de tenir (en tant que) parole.

Et c'est là la singularité de l'écriture de Natasha Kanapé Fontaine : puiser dans le trauma, le dire pour le guérir et parvenir, par cette expression même, à une parole nouvelle, souveraine, décolonisée, une parole désirante, une parole verticale. Dans ce contexte, la 
notion de désir n'est pas à prendre dans son sens libidinal ou psychanalytique, c'est aux travaux de la chercheuse Unangax Eve Tuck et à ce qu'elle nomme une « méthodologie du désir » que je fais référence :

This framework, what I would come to call a framework of desire, is aided by Deleuze theorizing of desire [...]. This framework is intent on convoking loss and oppression, but also wisdom, hope, and survivance (Tuck 2009 ; Vizenor 1998). Anishinaabe scholar Gerald Vizenor tells us that our storytelling that emphazises survivance is an 'active repudiation of dominance, tragedy, and victimry' and these survivance stories 'are the creases of transmotion and sovereignty' (1998, p. 15).

A desire-based research framework recognizes and actively seeks out complexity in lives and communities. It dismisses one-dimensional analyses of people, communities, and tribes as flattened, derelict, and ruined. Desire-based research frameworks appreciate that all of us possess a: 'complex and oftentimes contradictory humanity and subjectivity that is never adequately glimpsed by viewing [one another] as victims or, on the other hand, as superhuman agents' (Gordon 1996, p. 4 ; Tuck 2009)33. (Tuck, 2010, p. 639. L'auteure souligne.)

Bien que Eve Tuck ne soit pas une chercheuse dans le domaine littéraire, ce qu'elle nous dit ici a tout à voir avec les possibilités ouvertes par la littérature. En effet, la littérature n’a pas à choisir, à prendre position, à binariser. Plutôt, elle complique, complexifie, nuance, contredit et c'est là sa grande force. Également, je veux pousser plus loin la relation entre la conceptualisation du désir chez Tuck et celle de la survivance chez Gerald Vizenor. Or, il apparait important de noter que Eve Tuck établit déjà cette relation au cœur même de son

\footnotetext{
${ }^{3}$ « Ce cadre, que j'ai nommé une méthodologie du désir, est aidé par la théorisation deleuzienne du désir (...). Un tel cadre vise à convoquer les notions de perte et d'oppression, mais aussi la sagesse ou le savoir, l'espoir et la survivance. L'intellectuel Anishinaabe Gerald Vizenor écrit que les récits qui mettent l'accent sur la survivance opère une " répudiation active de la domination, de la tragédie et de la victimisation " et ces récits de survicance " sont les lieux à travers lesquels l'on peut saisir la transmotion et la souveraineté ". Une méthodologie fondée sur le désir reconnaît dès lors et cherche activement à mettre en lumière la complexité de la vie et des communautés. Elle rejette les analyses unidimensionnelles des individus et des communautés comme étant aplanies, abandonnées et ruinées. Les cadres de recherche fondés sur le désir reconnaissent que nous possédons tous: " une humanité et une subjectivité complexes et souvent contradictoires que l'on n'entrevoit jamais adéquatement en se considérant[les uns les autres] comme des victimes ou, d'autre part, comme des agents surhumains ". » (Je traduis.)
} 
MARIE-ÈVE BRADETTE, «Elles se relèvent : penser la résurgence dans la langue et la littérature Innues »

texte. Ce qu'elle récupère de la pensée de Vizenor concerne l'idée précise que les récits de survivance s'incarnent dans une pratique de la souveraineté à même la narration et que par ces récits les sujets en présence refusent le statut de victimes au profit d'une prise en compte de leur pouvoir sur le sens de leurs récits, ce que Scott Richard Lyons nommait une souveraineté rhétorique (2010), un pouvoir qui s'écrit non seulement dans le moment présent, mais dans une temporalité du devenir qui s'enrichit de ce qui la précède : « desire is about longing, about a present that is enriched by both the past and the future » (Tuck, 2010, p. 644).

On lira en ce sens, c'est-à-dire en tant qu'écriture du désir et de la survivance, une écriture à cet effet nettement décolonisatrice, les vers suivants du slam «L'âme en tannage » de Natasha Kanapé Fontaine :

je ne suis pas une peau à vendre une nation à suspendre sur le mur du salon je te le dis tout de suite : je ne resterai pas une crise d'oka enfermée dans un livre d'histoire de toute façon. et quand on me regardera on regardera un peuple uni et debout fort de la force du tonnerre ! mes chants de paix seront la sève de ma survivance fière ! (Kanapé Fontaine, cité par St-Amand, 2015, p. 1. Je souligne)

Dans ce passage, ce n'est plus seulement la femme qui se lève, mais le peuple Innu tout entier. Il se met debout afin de chanter sa survivance. De plus, dans la manière dont le texte se décline, le refus d'une histoire enfermée dans les traumatismes du passé — l'exemple de la crise d'Oka est parlant — et le désir de guérir le traumatisme pour accéder à la recréation, on peut lire les traces explicites d'une résurgence culturelle. Chez Manon Nolin, une autre écrivaine Innue dont le premier recueil de poésie, Ma peau aime le Nord, a été publié en 2016 
aux éditions Hannenorak, l'écriture du traumatisme est également palpable, elle y est même centrale, mais il n'y a pas encore un levier de recréation identitaire et politique pleinement assumé, un mouvement vers la décolonisation qui ne pourra s'enclencher qu'une fois les torts dénoncés. Toutefois, écrire le traumatisme et les affres du colonialisme est déjà, chez l'autrice originaire de Mingan, le premier pas vers le rétablissement d'une posture verticale qui apparait dans un poème en guise de demande, d'imploration adressée à la femme, l'ancêtre venue avant la poète :

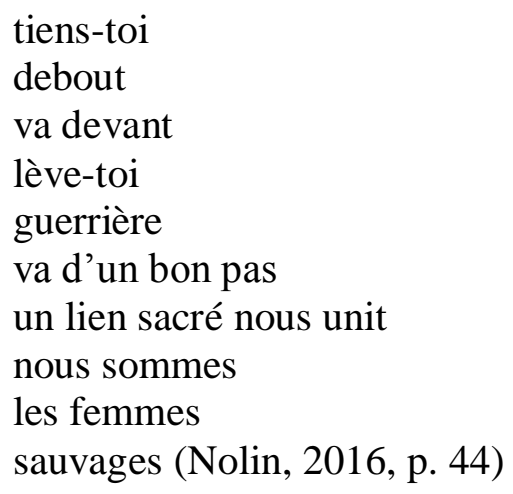

Pour poursuivre la réflexion au sujet d'une parole féminine et qui plus est de femme Innue, le court texte « Ma parole rouge sang », publié en 2015 dans la revue Relations s'avère révélateur, notamment en ce qui concerne les langues Innue et française, voire la poésie qui agit, dans l'écriture de Natasha Kanapé Fontaine, elle, comme un véritable levier de décolonisation, puis de souveraineté identitaire, féminine, politique, mais aussi orale et mémorielle (Marcoux, 2017, p. 69). Retrouver sa langue maternelle après l'avoir désapprise dans l'enfance, c'est, pour la poète, « un moyen incontournable pour devenir une femme » (Kanapé Fontaine, 2015, p. 25). Et si le féminin est lié à la langue dans cette énonciation, à la langue Innue dans ce texte en langue française, c'est que la langue maternelle réapprise, permet, seule peut-être, d'envisager cette figure littéraire singulière et puissante de la femme- 
MARIE-ÈVE BRADETTE, «Elles se relèvent : penser la résurgence dans la langue et la littérature Innues »

debout. En effet, bien que cette figure se donne à lire en français, il faut être sensible à la manière dont le français porte en lui l'innu-aïmun, la langue du territoire, la langue de celles d'avant. La parole de la poète se relève ainsi dans l'utilisation qu'elle choisit de faire de la langue coloniale démontrant comment les deux langues sont intimement liées dans l'élaboration du geste poétique :

le français sera mon arme de déconstruction massive contre le colonialisme, cette attitude outrancière rencontrée au quotidien. Cette arme affinera ma pensée, agrandira ma mémoire, elle émancipera mes opinions et ma parole. [...] La langue française sera mon arme de reconstruction massive. Ma parole aura la couleur de mon sang. (Kanapé Fontaine, 2015, p. 25)

Suivant cette disposition, on peut comprendre le travail de Natasha Kanapé Fontaine comme une littérature de la survivance. Dans Manifest Manners (1994), puis plus récemment dans Native Liberty (2008) le chercheur Anishinaabe Gerald Vizenor aborde ce qu'il a été parmi les premiers à définir comme une pratique de la survivance dont les actes se situent à même les ruines de la représentation coloniale (1994, p. 3), ce que l'on peut également nommer une archive coloniale. La survivance vise dès lors à faire advenir une littérature qui résiste à la dominance, à l'hégémonie répandue par le colonialisme en Amérique du Nord en fait. « Redonner vie aux ombres », pour reprendre les mots de Natasha Kanapé Fontaine cités plus haut, implique un geste de recréation, une renaissance, voire une relève, mais c'est surtout une manière de témoigner de la présence d'une continuité culturelle au contraire des seules ruines coloniales qui, elles, postulent l'effacement et l'absence de toute culture autochtone et avec elle de tout corps autochtone, puis de ses possibilités signifiantes. Pour Natasha Kanapé Fontaine, mais aussi pour Leanne Betasamosake Simpson, il y a une volonté de replacer le corps sur le territoire (réel comme imaginaire), dès lors d'écrire la présence comme 
résurgence. Le travail sur le langage permet, de plus, d'envisager avec acuité la résurgence au féminin : Une déconstruction, puis une reconstruction du sujet féminin, de la femmedebout en relation avec le territoire. Et si j'insiste sur la constitution d'un sujet féminin, d'une résurgence autochtone particulièrement féminine, c'est à la fois pour des raisons matérielles qui concernent la présence fulgurante des femmes autochtones dans le champ littéraire actuel, mais aussi pour montrer comment, en assumant cette posture debout, en replaçant le corps féminin sur le territoire littéraire, les autrices opèrent une véritable critique de la colonisation et de ses violences genrées. En effet, la colonisation s'est faite sur le corps féminin. Les femmes autochtones ont été désubjectivisées et déshumanisées (Maracle, 1996) dans une rhétorique et un agir coloniaux. Une colonisation qui, de surcroit, doit être envisagée dans ses aspects hétéropatriarcaux (Smith, 2011). Ainsi, par l'écriture littéraire, les femmes autochtones font acte de résistance dans un mouvement de re-féminisation et de décolonisation.

Or, et pour revenir aux textes, cette figure de la femme qui se relève dans la poésie n'est pas exclusive à la poète originaire de Pessamit, on la retrouve aussi chez Manon Nolin comme on l'a vu, mais j'y vois là plus encore une figure récurrente et absolument significative de la poésie Innue en général dès lors qu'elle se donne à lire comme un tissu conceptuel à déplier pour en donner à voir et à comprendre les différents pans. Chez MarieAndrée Gill, la femme-poète se dresse elle aussi. Elle le fait toutefois tranquillement, lentement ${ }^{4}$ dans un mouvement au ralenti qui est propre à son expression poétique, ce que

\footnotetext{
${ }^{4}$ Ici, je reprends des éléments discutés lors d'une conférence de Marie-Andrée Gill au $7^{\text {ième }}$ Salon du livre des Premières Nations présenté à Québec en novembre 2018. L'autrice se questionnait sur ce que cela signifie que de se lever calmement au contraire d'un mouvement militant ou activiste.
} 
MARIE-ÈVE BRADETTE, «Elles se relèvent : penser la résurgence dans la langue et la littérature Innues »

Jonathan Lamy, dans sa préface à Béante, qualifie «[d'une] esthétique de l'audace décomplexée » (Lamy, 2015, p. 9). En effet, alors que le sujet féminin chez Natasha Kanapé Fontaine se relève avec puissance, parfois accompagné du retentissement du tonnerre, dans un mouvement très souvent de revendication politique - en témoigne notamment ses slams territoriaux et Manifeste Assi, son recueil sans doute le plus engagé rédigé en droite filiation avec le mouvement militant Idle no more — ce que je nommerai une poétique de la relève est plus subtile dans la poésie de Marie-Andrée Gill. D’une subtilité dans ses liens à l'élément liquide, à la rivière, calme et dont les dessous s'agitent, affichant leur mouvement par de légères secousses à la surface. Une rivière si souvent mise en scène dans l'écriture de Marie-Andrée Gill.

\section{Maintenir la posture}

Ne s'exprimant pas à travers une plume revendicatrice, mais plutôt donnant à lire une écriture du quotidien — des jeux de langage inspirés des paroles d'enfants par exemple, comme dans Béante - Marie-Andrée Gill m'apparait être de ces écrivaines qui assurent le maintien de la posture verticale. Si la femme se relève au sein de l'écriture, elle reste, chez Gill dans cette position dans la mesure où l'écriture, justement, ne se fait pas militante, mais inscription du désir, lieu de son élaboration à même la quotidienneté. Le désir, je le définis ici dans ses rapports à la verticalité et, ce faisant, je construis mon argumentation à partir des travaux de Eve Tuck cité en début d'article, mais aussi de la représentation visuelle du désir dans la création artistique de l'artiste Anishinaabe Nadia Myre. En effet, l'œuvre Wood of Desire de Myre présente une installation faite de bout de bois dressés à la verticale et tous reliés par un mince fil rouge, dans un effort communautaire, en une solidarité manifeste dont la posture debout est nécessaire, certes, mais justement rendue possible par la collaboration avec les 
www.revue-analyses.org, vol. 14, nº1, printemps- été 2019, p. 98-123.

autres êtres humains, avec les éléments naturels également, ici le bois. À cet effet, on peut voir les concepts de désir, de survivance et de résurgence comme étant liés dans leur volonté de donner à entendre les voix de sujets pleinement décolonisés, de sujets individuels et de sujets collectifs tout à la fois.

Le travail poétique que poursuit Marie-Andrée Gill avec son recueil paru en 2015 permet subséquemment de déployer le cadre conceptuel mobilisé ici dans ses liens fondamentaux au corps et à la langue, à une langue littéraire qui se fait corps de désir et désir du corps tout à la fois. Un rapport au désir qui, dans la poésie de Marie-Andrée Gill, s’incarne aussi dans ses aspects féminins, voire maternels. Pour reprendre les mots de Kateri Akiwensie Damm, le désir serait alors créatif et procréatif (2016, p. 394). L’agentivité du désir tel qu'il se met en scène chez Marie-Andrée Gill est en effet liée à son agentivité de femme et de mère tel que la poète Ilnue le revendique dans une entrevue qu'elle accorde à David Sioui pour le journal de Wendake La griffe du Carcajou : «Je suis ilnue, je suis québécoise, je suis une femme, une mère et je suis plein d'autres choses » (Lamy, 2013, p. 37) . Avec cette affirmation où le $J e$ se répète et se dresse dans une prise de position subjective forte, MarieAndrée Gill refuse l'étiquette unique et sa poésie met cela en scène, en mots, en corps et en affects.

En ce sens, il y a dans Frayer un corps, un corps pluriel matérialisé dans la langue de l'écriture, corps et langue coïncidant, dans le travail de la poète, avec le territoire liquide formé par la rivière, une rivière réelle, mais une rivière imaginaire surtout, faite des mots de sa poésie, de ses mots à elle. Apparait à cet effet une véritable relation entre la langue, le texte et le corps dans la poésie. Relation qui se tisse par le recours à la figure de la ouananiche. J'ajouterais de plus, et en reprenant certains éléments d'analyse de l'article « Bodies, 
MARIE-ÈVE BRADETTE, «Elles se relèvent : penser la résurgence dans la langue et la littérature Innues »

Sovereignty, and Desire : Aboriginal Women's Writing of Québec » de Sarah Henzi, un article qui couvre la production littéraire de plusieurs autres écrivaines, qu'il y a, dans la performance poétique à l'œuvre dans l'écriture de Marie-Andrée Gill, une forme « d'intervention du corps qui explore l'intersection entre le texte et la sensualité » (Henzi, 2015, p. 86. traduction libre.).

La lectrice qui entre dans le texte se voit happée, dès les premières pages, par une proposition poétique forte, celle de « frayer / à même la cicatrice / frayer » (Gill, 2015, p. 9) et par la nécessité d'entrer dans ce texte lentement, sans ses chaussures peut-être ${ }^{5}$, pour ne pas risquer, précisément, de rouvrir la cicatrice. Celle qui se fait rupture et suture tout à la fois. La cicatrice est en effet un témoignage du passé, une inscription sur le corps d'une mémoire douloureuse ; elle est l'interstice à l'intérieur duquel nous convie la poète. Elle nous y convie, de surcroit, à l'aide d'un guide métaphorique qui agit comme une suture dans le texte, mais aussi entre le texte et le corps, entre le texte et la rivière de manière à ce que les deux se confondent dans l'espace poétique liquéfié. La ouananiche est ainsi convoquée dans de petits intermèdes entre les différentes sections du recueil de poésie. Le poisson dont le nom, « en ilnu [...] signifie : "Celui qui se trouve partout" ou "le petit égaré” » (Gill, 2015, p. 11), est une figure qui remonte le courant de la rivière textuelle, guide le lecteur de cicatrice en cicatrice. La manière dont le texte requiert la signification du mot, en Ilnu, donne d'ailleurs à lire une présence de la langue, un désir de la langue dans l'écriture alors que cette dernière ne se matérialise pas dans le grain du texte. Par cette non-coïncidence des langues, le texte revêt un caractère fondamentalement intermédiaire qui correspond à la fraie, c'est-à-dire à la

\footnotetext{
${ }^{5}$ Je paraphrase ici le titre d'un recueil de Natasha Kanapé Fontaine, N'entre pas dans mon âme avec tes chaussures, paru en 2012 aux éditions Mémoire d'encrier.
} 
www.revue-analyses.org, vol. 14, nº1, printemps- été 2019, p. 98-123.

ponte des œufs de poisson. Mais le mot « fraie » est aussi lié au verbe frayer du titre en ce qu'il implique un mouvement, un déplacement dans un espace exigu. C'est donc une manière pour la langue Ilnue de se frayer un chemin à même la langue française, à ses côtés, comme en une marche, côte à côte. Se frayer un chemin dans le texte, dans la vie, dans la langue et à la croisée des langues, dans un entre-deux poétique qui fait converger, à même l'écriture, les imaginaires Ilnu et québécois et, de ce fait, le geste de frayer remet en cause l'hégémonie des identités dans un acte de souveraineté et de désir.

Alors que le recueil ne présente à peu près aucune mention explicite d'une langue autochtone, cette dernière se retrouve pourtant partout dans le tissu textuel, dans l'énonciation comme dans les figures fantomales décrites, dans « les fantômes restés collés / sur la tempête de nos corps »(Gill, 2015, p. 19), dans un propos qui revient, fait retour, surgit et survit en se dressant, tel le désir pleinement réclamé (on se souvient des bâtons dressés dans l'œuvre Wood of Desire de Nadia Myre). La fraie, le fait de frayer, c'est donc aussi, pour reprendre l'expression de Marie-Andrée Gill dans une entrevue qu'elle accorde à la revue numérique Le crachoir de Flaubert en 2016, « occuper le territoire de la langue » (Gill, 2016). L'occuper, l'habiter dans et par le langage, dans et par le corps, dans un mouvement du temps également, c'est « lécher la surface de l'eau avec la langue que je / ne parle pas » (Gill, 2015, p. 24). Ces vers dévoilent une relation intime et même sensuelle entre la langue autochtone et la langue comme organe physiologique et permet d'entrevoir un devenir corps de la langue dans la poésie et donc une possibilité physique pour la langue de se lever, de prendre et de maintenir la posture verticale du corps. Devenir corps du lac également, car « le lac gruge un peu plus le ciment les gencives en sang (Gill, 2015, p. 14). C'est de manière inversée que se travaille le rapport entre le corps, la langue et l'eau ici. Le lac devient, 
MARIE-ÈVE BRADETTE, «Elles se relèvent : penser la résurgence dans la langue et la littérature Innues »

lui aussi, corps, il se matérialise dans des gencives en sang qui évoque, encore une fois, une forme d'intimité, de violence également, mais surtout de sensualité «comme ce premier french sur le rempart » (Gill, 2015, p. 14).

À ce lien entre la langue et l'élément liquide s'ajoute, dans les pages qui suivent, le vers suivant : « une seule chose tempère : l'eau douce » (Gill, 2015, p. 26). Comme si l'eau sur la langue pouvait tempérer, donner une autre forme, apaiser et réparer le sentiment d'une langue non parlée, spoliée, d'une langue léchant la surface de l'eau dans une proximité toute corporelle. À propos de l'écriture de Marie-Andrée Gill, l'on peut ainsi constater une résonnance et une résurgence de la langue, la mise en réseau et en écho dans le texte d'un champ sémantique lié à la langue et dont la figure de l'eau se fait lien, suture, cicatrice à même l'écriture.

Avec le désir inscrit dans le corps, devenant corps, un corps liquide, la temporalité du désir performée dans la poésie est aussi temps de la résurgence, tout entier tourné vers le futur, mais s'enrichissant du passé (Tuck, 2010, p. 644 ; Simpson, 2018, p. 120). La poésie est en ce sens le lieu où s'écrit et se corporalise le désir dans ses liens à l'avenir, à ce qui est encore à venir. Frayer s'ouvre en effet sur les mots suivants : « Nous autres les probables/les lendemains/les restes de cœur-muscle/et de terre noire/Nous autres en un mot : /territoire » (Gill, 2015, p. 7). Ces mots inscrivent la communauté dans une perspective du lendemain et de ses possibilités, mais en marquant cette temporalité d'un reste. Plus loin, on peut lire les vers suivants : «comment prédire autre chose/que des miracles croches/de toute façon » (Gill, 2015, p. 24). Nous sommes là confrontés à une impossibilité d'envisager le futur comme un espace de re-création identitaire, de réappropriation. Le futur, dans ce passage, s'ancre dans le passé sans le transcender. C'est plutôt dans un passage ultime que l'on voit 
www.revue-analyses.org, vol. 14, nº1, printemps- été 2019, p. 98-123.

poindre le désir d'un passé à reconstruire au futur : «Au milieu de la trajectoire/du bleu-gris des yeux du lac presque calé/il y a notre rêve : une femme debout/de tous ces hiversmondes/accumulés dans la glace à refaire »(Gill, 2015 , p. 75. Je souligne.). Le rêve est ici associé à l'acte de refaire, mais surtout à une posture revendiquée et assumée poétiquement, celle de la femme debout, de la femme qui résiste dans le langage au passé, qui l'assimile pour en faire l'objet d'une création, d'une procréation poétique nouvelle. Or, cet énoncé, je crois qu'il convient de le penser dans une forme performative en ce sens où l'énonciation devient l'accomplissement du désir, sa manifestation dans le texte. Performativité et poésie composent alors un couple indissociable. Lorsque la poète écrit « la femme debout », cette dernière, sujet même de l'écriture, se lève au cœur de la poésie, au cœur de l'écriture.

\section{Marcher vers l'avant}

Debout, la femme et avec elle la langue et la poésie se forment, en quelque sorte, comme les métaphores du sujet féminin, se mettent en marche et enclenchent un mouvement vers le futur décrit dans le recueil Frayer de Marie-Andrée Gill. Marcher vers l'avant est, dans cette perspective, un mouvement spatial et temporel tout à la fois les deux paradigmes se joignant à travers la trajectoire même d'une marche millénaire sur le territoire. À partir de la conjonction du temps et de l'espace, l'on peut, en outre, appréhender la notion de résurgence, puis l'idée d'une transmission transgénérationnelle qui y est sous-jacente, proposée dans cet article comme une alternative à la notion trop réductrice d'émergence littéraire. La marche est ainsi un mouvement vers le futur, mais en même temps tourné vers le passé ce qui fait en sorte que les deux temporalités se télescopent et s'aplanissent à même l'écriture et donne à penser, avec une acuité renouvelée, les termes d'une poétique de la relève en ce sens que l'acte de se relever implique une chute préalable, une attache nécessairement au passé, mais 
MARIE-ÈVE BRADETTE, «Elles se relèvent : penser la résurgence dans la langue et la littérature Innues »

dont le futur se fait lieu de resymbolisation, de réinterprétation et, particulièrement de recréation. Dans un article qu'elle consacre entièrement à la littérature autochtone dans le contexte francophone du Québec, Michèle Lacombe utilise la métaphore de la marche comme une manière d'aborder les «continuous and discontinuous movement between ancestral understandings based in Indigenous languages and cultures and the coexistence of such knowledge with European languages and urban cultures ${ }^{6} \gg$ (Lacombe, 2014, p. 159). Michèle Lacombe abordera ainsi la marche dans ses aspects formels, comme la métaphore par excellence du mouvement poétique. Elle écrit :

Words that walk - whether in referential, metaphorical, translated, spoken, or dialogical language - point in a number of different directions that are more or less accessible to the reader, depending on her or his experiences and language skills. Indigenous poetics allows for a wide range of linguistic, formal and rhetorical strategies, realigning the contemporary poetic voice with older forms of storytelling. For me, the rhythms of the poetic foot, sometimes moccasined and sometimes not — rhythms associated with the heartbeat, with human breath, and with dancing in Indigenous and other languages - are embodied in "words that walk"7. (Lacombe, 2014, p. 160).

À la suite de l'analyse proposée des poèmes de Marie-Andrée Gill, cette proposition selon laquelle les mots se matérialisent, se corporalisent dans et par le mouvement de la marche m'apparait absolument intéressante et surtout une manière originale de réfléchir à ce que peut

\footnotetext{
${ }^{6} \ll[\ldots]$ les mouvements continus et discontinus entre les savoirs ancestraux ancrés à même les langues et les cultures autochtones et la coexistence de ce savoir avec les langues européennes et les cultures urbaines. » (Je traduis.)

${ }^{7}$ «Les mots qui marchent - que ce soit en langage référentiel, métaphorique, en traduction, en parole ou en dialogue - pointent dans différentes directions qui sont plus ou moins accessibles au lecteur, selon ses expériences et ses compétences linguistiques. La poétique autochtone offre un large éventail de stratégies linguistiques, formelles et rhétoriques, réalignant la voix poétique contemporaine avec les formes narratives plus anciennes. Pour moi, les rythmes du pied poétique, tantôt chaussé de mocassins, tantôt non — des rythmes associés au battement du cœur, au souffle humain, à la danse dont les mouvements se dessinent dans les langues autochtones et dans d'autres langages — se matérialise dans des "mots qui se mettent en marche". » (Je traduis.)
} 
signifier se relever en poésie en ce sens que le corps est nécessairement impliqué dans cette action, dans le redressement. Par ailleurs, les expressions de l'espace et du temps incarnées dans la marche qui devient lieu de leur mise en scène à même le corps, les rassemblant en un geste singulier, se manifestent avec éloquence dans la poésie de Joséphine Bacon. Dans son premier recueil de poésie, Bâtons à message/Tshissinuatshitakana, on peut lire la marche comme une pratique transmise de génération en génération et dont l'écriture transpose les significations :

\author{
Les anciens \\ marchaient sans cesse \\ ils tiraient leurs traîneaux \\ sur la neige \\ et quand elle fondait, \\ ils naviguaient. \\ J'ai perdu la trace \\ de leur passage \\ vers la terre dénudée \\ sans guide \\ pour m'orienter. (Bacon, 2009, p. 22)
}

Alors que ce poème décrit la difficulté de transmettre l'héritage lorsque les traces sont disparues, recouvertes par l'histoire coloniale, le poème placé en conclusion du prologue de Bâtons à message/Tshissinuatshitakana propose une ouverture quant à la possibilité de transmettre avec ce même mouvement des pas :

Accompagne-moi pour faire marcher la parole, la parole voyage là où nous sommes, suivons les pistes des ancêtres pour ne pas nous égarer, parlons-nous... (Bacon, 2009, p. 8) 
MARIE-ÈVE BRADETTE, «Elles se relèvent : penser la résurgence dans la langue et la littérature Innues »

Lorsque la poète invite à suivre les pas des ancêtres, elle invite à marcher à même les traces matérielles du passé, mais à le faire en avançant, en se déplaçant, par le truchement des pas et de la parole (poétique), vers ce qui reste encore à venir. Le rapport transgénérationnel impliquant un héritage dont le sens se fait au présent et au futur, un legs qui est, chez Bacon, legs culturel, legs de la langue, de la littérature. La voix de Joséphine Bacon, dans le paysage littéraire autochtone du Québec résonne en effet comme une source de transmission dans la poésie des générations qui la suivent. À cet effet, Natasha Kanapé Fontaine dira de son amie et mentor «qu'elle décide plutôt de prendre la voix de celle qui guide, de celle qui tend la main, de celle qui transmet la philosophie des ancêtres » (Tardif, 2018). La jeune poète Innue insiste sur cette place singulière qu'occupe son aînée qui est reconnue comme une passeuse d'histoires, celle qui raconte, celle qui transmet, qui laisse en suspens en prévision de celles qui viendront après elle, la femme dont le «dos ressemble/à une montagne sacrée,/courbée d'avoir aimé/tant de fois » (Bacon, 2009, p. 64). Si l'écriture de Joséphine Bacon prend part à ce mouvement d'une relève littéraire, ou plutôt si elle met en place les éléments d'une poétique de la relève telle que je l'ai décrite jusqu'à présent, c'est toujours en direction de celles qui viendront, de celles qui se relèveront à leur tour pour reprendre la posture qui est déjà celle de l'enfant dans le takanukan ${ }^{8}$ :

«Quand un enfant vient au monde, il est déjà debout », me disait hier mon amie Joséphine Bacon.

Dans le territoire, les femmes accouchaient et repartaient dès le lendemain, le nouveau-né tenu bien droit par le takanukan sur leur dos. Elles continuaient à marcher avec le clan contre le vent vers le nord, ou vers unipek $^{u}$, la mer; c'est là que les familles se retrouvaient pendant l'été pour laisser se reposer les animaux de l'intérieur des terres. En quatre saisons, les Innus effectuaient un immense aller-retour entre les rives du Saint-Laurent et la Baie d'Ungava.

\footnotetext{
${ }^{8}$ Le takanukan est le porte-bébé traditionnel utilisé par les Innu.
} 
Joséphine est née en chemin comme tous les Innus avant elle, jusqu'à elle. Au début des années cinquante, alors qu'elle entrait dans L'enfance, le rythme millénaire de la marche était en train de se briser. Les Innus subissaient alors une transition douloureuse. On venait leur enlever leurs enfants afin de les élever à leur place dans les pensionnats catholiques. (Morali, dans Bacon, 2009, p. 133)

La poète, initiée à la marche millénaire dès l'enfance, mais confrontée aux conséquences du colonialisme également, porte ainsi en elle les souvenirs et ce sont ces traces mémorielles qu'elle communique aux générations d'écrivaines qui lui succèdent. La poésie de Joséphine Bacon se met en marche, bien droite. La femme au dos courbée avance sur le territoire des mots et se relève au cœur de l'écriture. Par ce geste, cette marche scripturaire, la poète travaille à la réalisation d'une résurgence culturelle et littéraire.

\section{Repenser le concept de résurgence avec la langue et la littérature Innue}

En conclusion, j'aimerais lier ensemble les différents concepts mobilisés tout au long de la réflexion à travers la lecture et l'analyse de la poésie Innue contemporaine, ainsi ceux de survivance, de désir, de résurgence et de relève, les tisser à partir d'un verbe en langue Innue. En réfléchissant ainsi à «la langue comme méthode » tel que nous y enjoint Leanne Betasamosake Simpson à laquelle je reviens maintenant, et sur la manière dont l'autrice aborde le concept de résurgence, le travail des écrivaines Innues m'apparait être lié à l'action promue par le verbe unikapau. Selon le dictionnaire Innu/Français dont la ressource grammaticale est source d'information primordiale pour la recherche en littérature autochtone, unikapau signifie se relever, mais plus spécifiquement, il faut comprendre ce mot de la manière suivante : «il (humain) se met debout, se relève; il (ours) se dresse sur ses pattes d'en arrière ». Cette utilisation du verbe animé et transitif décrit bien la poétique à 
MARIE-ÈVE BRADETTE, «Elles se relèvent : penser la résurgence dans la langue et la littérature Innues »

l'œuvre dans la littérature Innue contemporaine. Le unikapau en tant que stratégie littéraire insiste de ce fait sur la faculté de se relever, de se remettre debout, une faculté que possèdent à la fois l'humain et l'animal, mais qui se transpose, chez les autrices étudiées ici, dans la poésie. C'est en effet la poésie qui opère ce mouvement. De la même manière que Michèle Lacombe aborde la marche en tant que métaphore de l'écriture, mais surtout avec sa proposition de voir l'incarnation de la marche et donc sa matérialisation corporelle dans les mots des poètes, je propose ici que l'écriture des femmes Innues met en œuvre une véritable poétique de la relève dont le verbe unikapau nous donne à comprendre pleinement la signification. Pleinement, c'est-à-dire en embrassant les possibilités du mouvement, de l'action, de son agentivité. Si la femme se relève dans l'écriture, elle le fait par choix, elle le fait pour décoloniser, elle donne à lire sa force, sa puissance, sa résurgence. Mais ce n'est pas seule qu'elle se dresse sur ses jambes, elle le fait accompagné des femmes qui sont venues avant elle et de toutes celles qui viendront après, de celles qui ont marché et qui marcheront, de celles qui ont écrit et qui écriront (avec) le territoire.

\section{Bibliographie}

AKIWENSIE-DAMM, Kateri (2016), «Erotica, Indigenous style », dans Morra, Linda M., \& Reder, Deanna (dir.), Learn, teach, challenge: approaching indigenous literatures, Waterloo, Wilfrid Laurier University Press.

BACON, Joséphine (2009), Bâtons à message/Tshissinuatshitakana, Montréal, Mémoire d'encrier.

CARON, Jean-François (2012), « La Plume autochtone/émergence d'une littérature », Lettres Québécoises, vol. 147, p. 12-15. 
COUlthaRD, Glen Sean (2014), Red skin, white masks: rejecting the colonial politics of recognition, Minneapolis, University of Minnesota Press.

Coulthard, Glen Sean (2018), Peau rouge, masques blancs: contre la politique coloniale de la reconnaissance, Montréal, Lux Éditeur.

GILL, Marie-Andrée (2012), Béante. Chicoutimi, Peuplade.

GILL, Marie-Andrée (2015), Frayer. Chicoutimi, La Peuplade.

HENZI, Sarah (2015), « Bodies, Sovereignties, and Desire: Aboriginal Women's Writing of Québec », Québec Studies, vol. 59, p.85-106.

Innu-aimun.ca: language resources for Innu: Words, Consulté 15 janvier 2019, à l'adresse http://dictionnaire.innu-aimun.ca/Words

JUSTICE, Daniel Heath (2018), Why Indigenous Literatures Matter, Waterloo, Wilfrid Laurier University Press.

Kanape Fontaine, Natasha (2012), N'entre pas dans mon âme avec tes chaussures, Montréal, Mémoire d'encrier.

Kanape Fontaine, Natasha (2014), Manifeste Assi. Montréal, Mémoire d'encrier.

KAnAPE FontAine, Natasha (2015), « Ma parole rouge sang », Relations, $\mathrm{n}^{\circ}$ 77), p. 24-25.

KAnaPE Fontaine, Natasha (2016), Bleuets et abricots. Montréal, Mémoire d'encrier.

KANAPE FONTAINE, Natasha (2018), Nanimissuat/Île-tonnerre. Montréal, Mémoire d'encrier.

LACOMBE, Michèle (2014), " "Pimuteuat/Ils marchent/They Walk": A few observations on Indigenous poetry and poetics in French », dans Neal McLeaod (dir.), Indigenous Poetics in Canada. Wilfrid Laurier Univ. Press.

LAMY, Jonathan. (2013), «Quand la poésie amérindienne réinvente l'image de l'Indien », Temps Zéro, $\mathrm{n}^{\mathrm{o}} 7$.

LinCOLn, Kenneth (1985), Native American Renaissance, Univ of California Press.

LyONS, Scott Richards (2000), «Rhetorical Sovereignty: What Do American Indians Want from Writing? », College Composition and Communication, 51(3), p.447-68. 
MARIE-ÈVE BRADETTE, «Elles se relèvent : penser la résurgence dans la langue et la littérature Innues »

MARCOUX, Gabrielle (2017), «L’Âme en tannage de Natasha Kanapé Fontaine: Souveraineté orale, territoriale et mémorielle », Canadian Review of Comparative Literature/Revue Canadienne de Littérature Comparée, vol. 44, nº1, p.68-81.

MARACle, Lee (1996), I am Woman: A Native Perspective on Sociology and Feminism, Press Gang.

MorAli, Laure (2009), «Postface», Bâtons à message/Tshissinuatshitakana, Montréal, Mémoire d'encrier.

Nolin, Manon (2016), Ma peau aime le Nord, Wendake, Hannenorak.

SIMPSON, Leanne Betamosake (2011), Dancing on our turtle's back: stories of Nishnaabeg re-creation, resurgence and a new emergence, Winnipeg, Arbeiter Ring Publishing.

SiMPSON, Leanne Betamosake (2018), Danser sur le dos de notre tortue :La nouvelle émergence des Nishnaabeg, Montréal, Nota Bene, «Varia ».

SMITH, Andrea (2011), «Queer Theory and Native Studies : The Heteronormativity of Settler Colonialism », dans Qwo-Li Driskill, Chris Finley, Brian Joseph Gilley, and Scott Lauria Morgensen (ed.) Queer Indinegous Studies, Tucson, The University of Arizona Press.

St-Amand, Isabelle (2015), La crise d'Oka en récits: territoire, cinéma et littérature. Québec, Presses de l'Université Laval.

TARDIF, Dominic (8 septembre 2018), « La lumière de Joséphine Bacon », Le Devoir.

TucK, Eve (2009), « Suspending Damage: A Letter to Communities », Harvard Educational Review, vol.79 n 3, p. 409-428.

TucK, Eve (2010). «Breaking up with Deleuze: Desire and Valuing the Irreconcilable », International Journal of Qualitative Studies in Education (QSE), vol. 23, n5, p.635-650.

VIZENOR, Gerald (1994), Manifest manners: Postindian warriors of survivance. Wesleyan University Press.

VIZENOR, Gerald (2009), Native liberty: natural reason and cultural survivance. Lincoln, University of Nebraska Press.

\section{Résumé}

Depuis le début des années 2000, les écrivaines Innues se positionnent à l'avant-scène de la production littéraire du Québec. La littérature Innue est dès lors considérée comme prenant part à une véritable «émergence » littéraire. Cet article propose d'étudier les textes de plusieurs autrices de manière à en dégager un cadre conceptuel critique et ainsi réviser l'idée 
d'une émergence littéraire qui est inappropriée. Plutôt que de parler d'émergence, cet article propose une réflexion au sujet de la résurgence littéraire et culturelle et de ce qui se présente comme une poétique de la relève chez les écrivaines Innues contemporaines.

\begin{abstract}
Since the early 2000s, Innu women's writers have been at the forefront of Quebec's literary production. Innu literature is therefore considered to be part of a literary «emergence». This paper proposes to study the texts of several women's authors in order to identify a critical conceptual framework and thus revise the idea of an inappropriate literary emergence. Rather than talking about emergence, this article proposes a reflection on the literary and cultural resurgence and what can be thought of as a poetics of redress.
\end{abstract}

\title{
Notice biobibliographique
}

Marie-Eve Bradette est candidate au doctorat en littérature comparée à l'Université de Montréal. Dans sa thèse, elle aborde les théories et les épistémologies autochtones du langage avec les littératures autochtones contemporaines. En 2018, son article « Langue française ou langue autochtone ? Écriture et identité culturelle dans les littératures des Premières Nations » a été publié dans un numéro consacré à «La notion d'autochtonie » de la revue Captures. Elle est, en outre, l'autrice de compte-rendus pour la revue Canadian Literature et la responsable du comité scientifique de la revue de recherche interdisciplinaire en textes et médias Post-Scriptum. Elle a également présenté ses recherches sur les littératures autochtones dans le cadre de nombreux colloques, notamment dans le cadre du congrès annuel de l'ILSA (conjointement avec l'APFUCC à Régina en 2018, et en 2019 à Vancouver), de l'APLAQA en Nouvelle-Écosse (2018), ainsi que dans le cadre du 7e Salon du livre des Premières Nations, à Québec (2018). 